\title{
Citoyens policiers ? Pratiques et imaginaires civiques de la sécurité publique dans la France du second XIX ${ }^{\mathrm{e}}$ siècle
}

Citizen-policemen? Civic imaginaries and practices of public security in France during the second half of $19^{\text {th }}$ century

Bürger-Polizei? Staatsbürgerliche Praktiken und Vorstellungen von öffentlicher Sicherheit in Frankreich in der zweiten Hälfte des 19. Jahrhunderts

\section{Arnaud-Dominique Houte}

\section{OpenEdition}

\section{Journals}

Édition électronique

URL : http://journals.openedition.org/rh19/4825

DOI : $10.4000 /$ rh 19.4825

ISSN : $1777-5329$

Éditeur

La Société de 1848

Édition imprimée

Date de publication : 1 juillet 2015

Pagination : 99-116

ISSN : 1265-1354

Référence électronique

Arnaud-Dominique Houte, « Citoyens policiers ? Pratiques et imaginaires civiques de la sécurité publique dans la France du second XIXe siècle », Revue d'histoire du XIXe siècle [En ligne], 50 | 2015, mis en ligne le 01 juillet 2015, consulté le 20 avril 2019. URL : http://journals.openedition.org/rh19/4825 ; DOI : $10.4000 /$ rh 19.4825 


\section{ARNAUD-DOMINIQUE HOUTE}

\section{Citoyens policiers? Pratiques et imaginaires civiques de la sécurité publique dans la France du second XIX ${ }^{e}$ siècle}

La scène se déroule peu avant 1914, dans un quartier calme de la capitale, à l'angle de la rue Rodier et de l'avenue Trudaine : deux jeunes gens aux allures d'apaches remontent le trottoir en bousculant les passants. Après avoir renversé l'étal d'un libraire, ils s'en prennent à un Juif, puis chapardent une brioche. "Personne dans la rue n'avait fait mine de s'apercevoir de rien. Les gens qui allaient à leur travail continuaient à descendre; un bistrot continuait à frotter le marbre d'une table qu'il avait poussée au bord du ruisseau ". Les voyous poursuivent leur chemin en se jetant des pommes de terre à la figure : "des passants en reçoivent, par ricochet, mais ne disent rien. Même un ou deux affectent de sourire, d'un air lâche». Et le narrateur de s'étonner : «Moi je pensais : c'est tout de même drôle que ça puisse se passer en plein jour. Où sont les agents?»"

Par cette description de l'indifférence coupable des badauds, par cette recherche spontanée du policier absent, cette nouvelle de Jules Romains entre en résonance avec l'imaginaire contemporain du fait divers, qui stigmatise la solitude et l'anonymat des "jungles urbaines" pour mieux l'opposer au temps jadis : "On est frappé, quand on lit les faits divers d'il y a un demi-siècle, par la grande solidarité en actes d'une société qui croyait en elle-même, par la facilité avec laquelle les braves gens risquaient leur vie pour d'autres braves gens face au crime», lit-on dans Le Quotidien de Paris en 1979; " aujourd'hui, chacun ou presque est persuadé qu'attaqué nul ne viendra à son secours $»^{2}$. D’Orange Mécanique (Stanley Kubrick, 1971) à 38 témoins (Lucas Belvaux, 2012) ${ }^{3}$, l'idée irrigue la fiction et nourrit le débat, alors même que se répand l'injonction sécuritaire : à l'heure des "voisins vigilants", le passager du métro apprend qu'il faut se montrer «attentifs ensemble ${ }^{4} \ldots$

1. Jules Romains, Sur les quais de la Villette, Paris, Figuière, 1914, p. 172-175.

2. Dominique Jamet, «Les assassins sont parmi nous», Le Quotidien de Paris, 9 décembre 1979.

3. Inspiré du roman de Didier Decoin, Est-ce ainsi que les femmes meurent? (Grasset, 2009), ce film met en scène les atermoiements des témoins silencieux d'un meurtre commis sous leurs fenêtres. 2013.

4. Jérôme Thorel, Attentifs ensemble! L'injonction au bonheur sécuritaire, Paris, La Découverte, 
Ce détour par l'actualité invite l'historien du XIX e siècle à reprendre les dossiers policiers avec un regard différent, non pour rechercher d'incertains échos du présent, mais pour mieux mettre en perspective une histoire de la sécurité publique qui s'est longtemps confondue avec l'histoire de la montée en puissance de la gendarmerie nationale ou des polices municipales, fers de lance de l'imposition d'un même modèle professionnel progressivement déployé sur l'ensemble du territoire. "À trop vouloir fonder une histoire de l'ordre public sur les corps de police qui le mettent en œuvre, on en oublierait ses fondements populaires", rappelle pourtant Pierre Prétou, qui plaide pour une réévaluation de la "clameur publique» et de la participation sociale aux actions de justice . La question se pose avec une acuité particulière pour la seconde moitié du XIX ${ }^{\mathrm{e}}$ siècle, dont plusieurs recherches récentes ont montré qu'elle constituait un point de bascule à partir duquel émergent des forces de l'ordre plus nombreuses, mieux réglementées, plus sûres d'elles-mêmes et de leurs armes ${ }^{6}$. Dès les années $1840^{7}$, l'idée s'impose que la police est une affaire de professionnels, et le déclin de la garde nationale scelle un nouvel âge de la sécurité publique, dont nous restons les héritiers, et qui contraste sensiblement avec le foisonnement des systèmes policiers du XVIII ${ }^{e}$ siècle et de la Révolution ${ }^{8}$.

Est-ce à dire que les pratiques anciennes ont disparu, que les citoyens ont renoncé à faire leur police? Évidemment non, comme le suggère, du reste, le dénouement de la nouvelle de Jules Romains par laquelle nous ouvrions cet article : «la rue, brusquement, avait sauté sur ces deux apaches, comme ça... comme si je serrais les mains d'un coup sur la gorge d'un poulet. Il n'y avait pas eu un mot, pas un signe. En un clin d'œil, la rue s'était crispée sur eux. Couic! Des deux trottoirs, les gens avaient bondi »". À moins qu'elles ne dégénèrent dans la violence et le lynchage, ces formes de police spontanée s'inscrivent rarement dans les archives. Il n'en est pas moins possible d'en rechercher la trace dans la littérature et les faits divers ${ }^{10}$, mais aussi au détour des dossiers de procédure judiciaire, emplis de passants qui viennent

5. Pierre Prétou, «Éléments pour une histoire de la clameur publique», in Frédéric Chauvaud et Pierre Prétou [dir], Clameur publique et émotions judiciaires de l'Antiquité à nos jours, Rennes, PUR, 2013, p. 22.

6. Quentin Deluermoz, Policiers dans la ville : la construction d'un ordre public à Paris, 18541914, Paris, Publications de la Sorbonne, 2012; Arnaud-Dominique Houte, Le métier de gendarme au XIX' siècle, Rennes, PUR, 2010; Aurélien Lignereux, La France rébellionnaire. Les résistances à la gendarmerie, 1800-1859, Rennes, PUR, 2008.

7. Fabien Gaveau, «De la sûreté des campagnes. Police rurale et demandes d'ordre en France dans la première moitié du XIX siècle", Crime, Histoire \& Société, 2000-4, p. 53-76.

8. Cet article a bénéficié des échanges scientifiques du programme SYSPOE (Systèmes policiers européens, XVIII'-XIX ${ }^{e}$ siècles) sélectionné par le programme blanc de l'ANR en 2012 (ANR12-BSH3-0004).

9. Jules Romains, Sur les quais de la Villette..., op. cit., p. 177.

10. À la fois des grands quotidiens, dont on connaît l'intérêt et qui bénéficient des chantiers de numérisation engagés par la BNF, et d'une presse locale attentive aux plus petits faits divers; AnneClaude Ambroise-Rendu, Petits récits des désordres ordinaires. Les faits divers dans la presse française de la Troisième République à la Grande Guerre, Paris, Seli Arslan, 2004; Dominique Kalifa, L'encre et le sang. Récits de crimes et société à la Belle Époque, Paris, Fayard, 1995. 
secourir les victimes et livrer des criminels ou des informations, voire dans certaines sources administratives, où perce une demande de reconnaissance d'actes héroïques.

Avouons d'emblée qu'il ne s'agira pas ici de faire l'inventaire de ces formes d'action, ni d'en mesurer l'évolution - tâche vouée à l'échec - mais d'en éclairer les logiques, tant il est vrai qu'il n'y a rien d'évident à intervenir sur la scène du délit ${ }^{11}$. Pourquoi se mêle-t-on de police, au risque du mauvais coup? Est-il possible d'identifier des "citoyens-policiers", héritiers des «citoyens-combattants» du premier XIX ${ }^{e}$ siècle ${ }^{12}$ ? Pour s'orienter à travers les réalités sociales et les représentations de cette sécurité informelle, on s'arrêtera sur quelques figures éclairantes : à la suite de Dominique Kalifa, on postulera en effet que l'imaginaire social se structure à travers "des intrigues qui lui donnent forme et sens» et dont l'historien peut reconstituer la trame ${ }^{13}$. Des Sherlock Holmes amateurs aux lyncheurs de la Belle Époque en passant par de "bons voisins", cette promenade ne prétend donc pas épuiser la description des pratiques; elle espère en faire mieux apparaitre la complexité.

$*$

\section{«Adieu L’ennui »: Les PASSIONS POLICIÈRES}

Héros de L'Affaire Lerouge, le père Tabaret est un vieux rentier solitaire qui a épuisé les plaisirs de l'étude. Aussi se jette-t-il dans l'art de l'investigation, dont il propose un vibrant éloge :

«Adieu l'ennui! Depuis que j'ai abandonné la poursuite du bouquin pour celle de mon semblable... ah c'est une belle chose! Je hausse les épaules quand je vois un jobard payer $25 \mathrm{~F}$ le droit de tirer un lièvre. La belle prise! Parlez-moi de la chasse à l'homme! Celle-là au moins met toutes les facultés en jeu, et la victoire n'est pas sans gloire. Là, le gibier vaut le chasseur; il a, comme lui, l'intelligence, la force, la ruse; les armes sont presque égales. Ah! Si on connaissait les émotions de ces parties de cache-cache qui se jouent entre le criminel et l'agent de sûreté, tout le monde irait demander du service rue de Jérusalem ${ }^{14}$.

On le sait, le personnage du détective bénévole est un archétype de la littérature policière naissante en cette seconde moitié du XIX siècle $^{15}$. Plus

11. Déborah Cohen, "Sur la scène du délit dans le Paris populaire du XVIII siècle : témoin ou acteur?", in Benoît Garnot [dir], Les témoins devant la justice. Une histoire des statuts et des comportements, Rennes, PUR, 2003, p. 333-341.

12. Louis Hincker, Citoyens-combattants à Paris, 1848-1851, Lille, Presses du Septentrion, 2008, notamment p. 117.

13. Dominique Kalifa, Les Bas-Fonds. Histoire d'un imaginaire, Paris, Seuil, 2013, p. 20-21.

14. Émile Gaboriau, L'Affaire Lerouge, $1^{\text {re }}$ éd. 1866, Paris, Le Masque, 2004, p. 47.

15. Elsa Lavergne, La naissance du roman policier français. Du Second Empire à la Première Guerre mondiale, Paris, Classiques Garnier, 2009, p. 78-81. 
riche que celui du policier, il libère la plume de l'écrivain et lui permet d'introduire une seconde trame narrative qui se tresse avec celle de l'enquête à proprement parler. Il s'inscrit, de plus, dans l'héritage romantique du redresseur de torts et constitue ainsi un chainon entre la figure du justicier, personnellement impliqué dans l'affaire, et celle de l'enquêteur moderne, qui n'agit que pour le plaisir de l'investigation. Aussi les premiers romans policiers proposent-ils de foisonnantes galeries de personnages, à la manière du Décapitée! de Fortuné de Boisgobey, où se mêlent un jeune fonctionnaire amoureux, un officier au fort caractère, un peintre astucieux, un vieillard scrupuleux - chaque protagoniste concourant à l'identification du coupable. Tous se démarquent pourtant avec dégoût des inspecteurs de police : "Je n'en suis pas, moi! Et je ne veux pas aider ceux qui en sont", s'écrie le brave pêcheur dont le témoignage sera décisif ${ }^{16}$.

Cette répugnance à l'égard de la police prend une forme encore plus marquée dans Le coup d'ceil de Monsieur Piédouche (1883). Enquêteur passionné et prétentieux, le personnage éponyme ressemble par certains aspects au père Tabaret. Policier amateur par goût autant que par appât du gain, il finit du reste par s'établir détective aux États-Unis, "profession très honorée là-bas", après s'être déconsidéré en France. Le vrai héros positif du roman est donc un jeune noble, Edmond de Chémazé, qui n'entre dans l'investigation qu’à contrecœur, pour protéger l'honneur menacé de sa famille. "Lui qui n'avait pas le génie policier» parvient pourtant à mener un interrogatoire en bonne et due forme, exige un aveu, prononce "une garde à vue» - le vocabulaire est éloquent, mais Chémazé reste fidèle à ses valeurs et laisse fuir la coupable, une fois celle-ci démasquée. Où l'on retrouve une morale exprimée dès le début du roman, alors que les protagonistes s'étaient mis à la poursuite d'une ombre inquiétante : "un gentleman peut sans déchoir remettre entre les mains de l'autorité un chenapan qui lui est tombé sous la main, mais ce n'est pas son métier de courir après lui, et il ne saurait le faire sans paraître grotesque. $»^{17}$

Cette littérature fait pourtant du citoyen le seul vrai limier, à la fois plus digne mais aussi plus efficace : «Moi, conscrit volontaire, j'avais vu clair là où le vieux routier de la sûreté s'égarait à tâtons», se réjouit le narrateur du Petit Vieux des Batignolles (Émile Gaboriau, 1876) qui empêche une erreur judiciaire ${ }^{18}$. Par ses méthodes plus inventives, mais aussi par sa meilleure connaissance du monde extérieur - et notamment de la bonne société bourgeoise - dont il déchiffre mieux les signes ${ }^{19}$, le détective amateur incarne un nouvel archétype social qui modèle les imaginaires et inspire des émules.

16. Fortuné de Boisgobey, Décapitée!, 1 1 éd. 1888, Paris, Les Belles Lettres, 2004, p. 226.

17. Fortuné de Boisgobey, Le coup d'ail de Monsieur Piédouche, Paris, Rouff, 1883, p. 318, 293 295,390 et 19 .

18. Émile Gaboriau, Le Petit Vieux des Batignolles, $1^{\text {re }}$ éd. 1876, Paris, Liana Levi, 1991, p. 93.

19. Manuel Charpy, «Enquêtes en chambre. Romans policiers et culture matérielle domestique 
Parmi les plus célèbres, le juge Magnaud, président du tribunal de Château-Thierry, n'hésite pas à se rendre aux courses de Longchamp, en 1890, "avec le projet très arrêté de se rendre compte par lui-même des procédés dont usent ces fameux pickpockets insaisissables pour la police de la capitale». Rendant compte des arrestations qu'il a ainsi menées à bien, la presse coule son récit dans le moule de la littérature policière; la chronique se teinte d'ironie quand elle s'interroge sur cet étrange "loisir de magistrat», mais elle rend hommage à la sagacité de celui qui «opère avec plus de succès que les agents de M. Lozé», alors préfet de police ${ }^{20}$. Encore quelques années, et vient le temps du "sherlockisme" : "Sherlock Holmes n'aurait pas fait mieux", titre ainsi Le Matin à propos d'un modeste courtier en bijoux qui, dupé par un intermédiaire véreux, parvient à remonter les filières internationales du recel et à démasquer des trafiquants viennois ${ }^{21}$... Montés en épingle par une presse gourmande de belles histoires, ces exemples isolés sont la face émergée d'une fascination criminelle bien partagée.

La démocratisation de ces passions policières se mesure à l'aune du courrier reçu par la sûreté de la préfecture de police lors des grandes affaires criminelles. Le fait est déjà signalé par Maxime Du Camp à la fin du Second Empire : «dès qu'un crime est commis, il se trouve de bonnes gens inoccupés qui se mettent l'esprit à la torture pour découvrir quel peut en être l'auteur, et les lettres pleuvent dru comme grêle», à tel point que plusieurs agents, les "écosseurs", sont chargés de les dépouiller ${ }^{22}$. Mais il se développe surtout à la fin du siècle : combien de dénonciations plus ou moins farfelues prétendent dissiper les mystères de l'affaire Steinheil ou retrouver la Joconde disparue? "La plupart de nos lecteurs semblent croire que nous avons organisé un concours et qu'il s'agit de deviner où est la Joconde» regrette Baschet, directeur de L'Illustration, qui fait face à un afflux de courriers ${ }^{23}$. Et que de révélations au sujet du crime de Soleilland : «je crois que je suis sur la bonne voie» annonce "un lecteur assidu du journal» qui se lance sur la piste d'une filière de traite des blanches, tandis qu'un autre pense retrouver la trace de la victime du côté de Verdun - et se plaint de n’être pas écouté par le «gros gendarme» auquel il s'est adressé24 !

Relégués au fond des boîtes d'archives, ces lettres anonymes et ces délires mythomanes souvent répétitifs mais rarement prévisibles suscitent désormais l'intérêt de l'historien ${ }^{25}$. Au-delà des modes médiatiques dont ils offrent un

au XIXe siècle» in Marta Caraion [dir.], Usages de l'objet. Littérature, histoire, arts et techniques, XIX'eXXe siècles, Seyssel, Champ Vallon, 2014.

20. «Loisir de magistrat», Le XIXe siècle, 11 décembre 1890. L'affaire fait l'objet d'une importante revue de presse conservée dans le copieux dossier personnel du juge Magnaud; Arch.nat. BB6 II 1039.

21. Le Matin, 30 avril 1913.

22. Maxime Du Camp, "La préfecture de police et la sûreté publique à Paris», Revue des Deux Mondes, juillet 1869, p. 180.

23. Jean-Yves Le Naour, Qui a volé la Joconde?, Paris, Vendémiaire, 2013, p. 55-58.

24. Cité par Jean-Marc Berlière, Le crime de Soleilland, les journalistes et l'assassin, Paris, Tallandier, 2003, p. 173-177.

25. Philippe Artières, "Archives du corps, archives de la biopolitique», Cahiers d'histoire, 2012, 
écho assourdi, ils témoignent en effet de la fascination croissante pour les faits divers et de la vulgarisation de l'enquête criminelle, dont tout un chacun semble connaître et adopter les principes ${ }^{26}$. Par leurs justifications embarrassées («je n’ai pas le droit de vous cacher ce que j’ai découvert», etc.), ils révèlent aussi la prise de conscience d'un devoir civique dont il faut interroger les fondements.

\section{ENTRE DÉLATION ET CIVISME}

La loi prévoit l'action policière du citoyen : «Tout dépositaire de la force publique et même toute personne sera tenu de saisir le prévenu surpris en flagrant délit", précise l'article 106 du Code d'instruction criminelle de 1808. Mais elle n'en fait pas une obligation ${ }^{27}$, au grand dam de certains juristes qui défendent l'introduction d'un véritable "devoir de dénonciation» sanctionné par une "astreinte pénale» ${ }^{28}$. "Dépourvu presque de tout droit en ce qui concerne une coopération désintéressée à la poursuite criminelle, le citoyen français n'est pas habitué à prêter main forte aux agents de l'autorité pour le maintien de l'ordre public", regrette encore Paul Nourrisson, en $1901^{29}$.

L'arrestation de Ravachol, au printemps 1892, constitue pourtant un moment décisif dans cette histoire de la collaboration citoyenne. C'est dans le journal qu'un garçon de café, Jules Lhérot, découvre le signalement de l'anarchiste. Le reconnaissant parmi ses clients, il fait prévenir la police, qui réussit à l'arrêter. Félicité, dessiné, photographié, célébré, le bien nommé Lhérot est au centre d'une agitation qui ne semble pas lui déplaire, mais qui suscite jalousies et menaces ${ }^{30}$. On connaît la suite de l'histoire ${ }^{31}$, l'attentat anarchiste qui frappe le restaurant Véry alors que s'ouvre le procès Ravachol, l'extrême émotion qui gagne la société, la fuite de Lhérot qui recherche désormais un anonymat protecteur...

Par les réactions qu'il a suscitées, l'épisode illustre les risques et les ambivalences de la dénonciation civique. Il alimente d'abord un discours critique dont on extraira le sévère jugement de Bernard Lazare : «quand une société

p. $75-90$.

26. Dominique Kalifa, L'encre et le sang..., op. cit., p. 286-291.

27. Exception faite du cas très particulier des "crimes projetés contre la sûreté intérieure ou extérieure de l'État», précise l'article 103 du Code Pénal de 1810. Ce n'est qu'avec la loi du 25 octobre 1941, puis l'ordonnance du 25 juin 1945 relative au «concours des citoyens à la justice et à la sécurité publique», qu'apparaît le délit de non-assistance à personne en danger.

28. Arnould Bonneville de Marsangy, De l'amélioration de la loi criminelle en vue d'une justice plus prompte, plus efficace, plus généreuse et plus moralisante, Paris, Cosse et Marchal, 1854.

29. Paul Nourrisson, L'Association contre le crime, Paris, Librairie de la société du recueil général des lois et décrets, 1901, p. 24.

30. Les proches de Lhérot ont d'ailleurs pris soin de déménager; Le Figaro, ${ }^{\mathrm{er}}$ avril 1892; Le Petit Journal, 26 avril 1892. p. 215 .

31. Jean Maitron, Le mouvement anarchiste en France, I, des origines à 1914, Paris, Maspéro, 1983, 
en arrive à glorifier la plus abjecte des fonctions, cette société est morte». Citons encore Adolphe Tabarant, qui reconnaît dans la foule admirative de «l'Iscariote Lhérot» (relevons la référence biblique) «le public des jours de guillotine": "ce pontife de la lâcheté souriait de temps à autre à des gueules de préfecture apostées au dehors pour sa sauvegarde, tandis qu'en un coin, des types à face pédérastique suivaient d'un regard brillant le jeune sauveur dont ils semblaient apprécier les formes ${ }^{32}$. En cet âge de virilité triomphante, la disqualification du «héros" par la féminisation constitue un procédé classique qui frappe de la même manière les très peu respectées «fileuses» - en argot, des individus «qui suivent les voleurs à la piste et, le coup fait, interviennent pour prélever un impôt fondé sur la crainte d'une dénonciation $»^{33}$.

Comme le rappellent ces exemples, la figure du dénonciateur suscite un malaise profond, ancien et largement partagé qui s'aggrave dès que s'y mêle la question d'argent - sans parler des enjeux politiques qui alimentent d'autres réflexes répulsifs ${ }^{34}$. Les archives restent par conséquent discrètes sur l'emploi des «mouchards» dont l'existence est pourtant attestée par l'attribution de fonds secrets - «le fléau de la délation salariée par l'État», disait le baron Méchin lors de l'un des rares débats publics organisés à ce sujet ${ }^{35}$. Les mémoires de commissaires hésitent sur le sujet : à la suite de Vidocq, matrice des discours policiers du XIX ${ }^{\mathrm{e}}$ siècle, Canler considère les indicateurs comme "des êtres misérables arrachés au crime par la crainte et vendant à la police pour une faible rétribution les secrets de leurs camarades ${ }^{36}$. Il n'en achète pas moins leurs services, de même que le font ses successeurs, Goron ou Rossignol, qui reconnaissent de la même manière les limites de la vénalité37.

Ainsi s'explique la position ambiguë du Petit Journal: "Si nous étions en Amérique, le gouvernement ferait donner une trentaine de mille francs à ce Lhérot qui a provoqué l'arrestation d'un aussi dangereux criminel. Nos moeurs plus chevaleresques s'opposent à ce système de primes à la délation. C'est bien fâcheux pour la sécurité publique ${ }^{38}$. Les mécanismes de rétribution existent cependant, en particulier pour ceux qui contribuent à attraper

32. Bernard Lazare, "Notes et notules», Entretiens politiques et littéraires, avril 1892; Adolphe Tabarant, L'En-Dehors, 10 avril 1892. Cité par Philippe Oriol, "Ravachol, saint et martyr», in Alain Pessin et Patrice Terrone [dir], Littérature et anarchie, Toulouse, Presses universitaires du Mirail, 1998, p. $125-128$.

33. Emile Colombey, Le monde des voleurs, leur esprit et leur langue, Paris, Hetzel-Dentu, 1862, p. 297.

34. Quelques perspectives historiques dans Christophe Cornevin, Les Indics, cette France de l'ombre qui informe l'Etat, Paris, Flammarion, 2011.

35. Pierre Karila-Cohen, «Les fonds secrets ou la méfiance légitime», Revue Historique, 2005-4, p. 731-766.

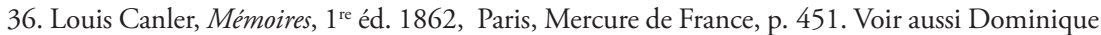
Kalifa, Crime et culture au XIX ${ }^{e}$ siècle, Paris, Perrin, 2005.

37. Laurent Lopez, «La Belle Époque du vol dans le ressort de la préfecture de police. Voleurs de Paris et voleurs de banlieue sous l'œil des forces de l'ordre", in Frédéric Chauvaud et ArnaudDominique Houte [dir], Au voleur! Images et représentations du vol dans la France contemporaine, Paris, Publications de la Sorbonne, 2014, p. 260.

38. Le Petit Journal, 31 mars 1892. 
les détenus évadés, mais ils restent plutôt confidentiels - pour ne pas dire honteu ${ }^{39}$. Des récompenses sont également promises, à la fin du XIX ${ }^{\mathrm{e}}$ siècle, par les agences de police privée ${ }^{40}$ et surtout par la presse, qui multiplie les promesses de primes à des fins manifestement publicitaires : L'Illustration offre jusqu'à $40000 \mathrm{~F}$ à celui qui ramènera la Joconde - et $10000 \mathrm{~F}$ pour tout renseignement décisif ${ }^{41}$.

Comme elle le fait avec Lhérot, la presse préfère néanmoins décerner le prestige de la notoriété à ces «bons citoyens" qu'elle érige en modèles. Ainsi Le Matin met-il à l'honneur, en 1909, le "valeureux» Joseph Lechthaler : âgé de 76 ans, ce rentier réussit en effet à arrêter les assassins de Jully, coupables d'un crime atroce qui a terrorisé la région. Aussitôt proposé pour une médaille d'honneur, il est photographié et immortalisé sur une carte postale, fusil en main; il fait surtout la couverture du supplément illustré du Petit Journal, qui vante le courage du vieillard : "Halte-là ou je tire "42. Le même hebdomadaire vante la bravoure des jeunes Maurice et Léon Laporte, qui ont su défendre le commerce paternel contre une tentative de vol. De cette affaire banale, dont le reste de la presse ne dit d'ailleurs pas le moindre mot, Le Petit Journal entend faire un événement et une leçon de moralité : «Dame! Nous nous sommes défendus, quoi!», explique avec une verve toute faubourienne le jeune héros; "nous avons vengé la pauvre vieille marchande qui vend des frites à côté et que ces misérables venaient de dévaliser avant d'entrer chez nous, et aussi une petite fille à laquelle ils avaient volé les deux sous destinés à son déjeuner $»^{43}$.

Attribuée par le ministre de l'Intérieur sur proposition préfectorale, la médaille d'honneur institutionnalise de la même manière la figure du "citoyen-sauveteur " ${ }^{44}$, et non celle du citoyen-policier. Cette décoration convoitée récompense en effet la lutte contre l'incendie, la noyade ou les animaux emballés; elle ne profite qu'à la marge à ceux qui ont su mettre des malfaiteurs hors d'état de nuire. Selon les estimations de Frédéric Caillé, confirmées par notre propre échantillon ${ }^{45}$, ces arrestations courageuses

39. Une prime de $20 \mathrm{~F}$ est versée selon le règlement général du 10 avril 1869 pour les colonies et maisons pénitentiaires de jeunes détenus, cité dans le Dictionnaire des connaissances générales utiles à la gendarmerie (Limoges, Lavauzelle, 1895, p. 691). Voir aussi H.-R. Lebrun, Manuel de police judiciaire, Paris, Rousseau, 1907, chapitre 5. On sait également que les évasions de bagnards sont signalées au public par un coup de canon : s'agit-il de prévenir les voisins du danger ou de les mobiliser? Voir Grégoire Chamayou, Les Chasses à l'homme, Paris, La Fabrique, 2010, p. 137.

40. "Cent francs de récompense" promet l'agence Goron à ceux qui donneraient des renseignements sur une jeune femme disparue; Archives de la préfecture de police, DB 40, prospectus sans date.

41. L'Illustration, 24 août 1911.

42. Le Matin, 15 décembre 1909; Le Petit Journal, supplément illustré, 26 décembre 1909.

43. Le Petit Journal, supplément illustré, 15 octobre 1905; Le Petit Journal, 2 et 3 octobre 1905.

44. Frédéric Caillé, La Figure du sauveteur. Naissance du citoyen secoureur en France, 1780-1914, Rennes, PUR, 2006.

45. Le relevé des dossiers (Arch.nat. sous-série F1dIII) a été entrepris pour les années 1858 (3 cas identifiés, sans tenir compte des professionnels de l'ordre, policiers, gendarmes, etc., qui peuvent également être récompensés), 1863 (6), 1878 (13) et 1893 (23). Même en tenant compte du doublement des affaires instruites, la progression reste évidente. 
restent rares (une affaire sur trente) jusqu’aux années 1880. Quand elles sont mentionnées, c'est souvent pour enrichir un dossier déjà chargé d'autres titres de gloire ${ }^{46}$. À la fin du siècle, on remarque néanmoins une meilleure reconnaissance de la participation citoyenne aux affaires de police. En profitent principalement des notables (des maires, un directeur de journal, un curé) et beaucoup d'employés publics (du facteur au gardien de square) qui savent sans doute mieux faire valoir leurs droits et constituer des dossiers de demande où s'exprime parfois - exagéré pour les besoins de la médaille? - le traumatisme du héros : «je dois vous dire aussi qu’à la suite de la scène qui s'est passée entre cet homme et moi j'en ai ressenti une telle impression que j'en ai fait une maladie», avoue ainsi un cultivateur qui a empêché la fuite d'un assassin $\operatorname{armé}^{47}$.

La récompense s'accompagne de félicitations publiques et d'encouragements au civisme. Elle remplit à cet égard une fonction pédagogique qui est clairement défendue par le sous-préfet de Mauléon, en 1863 : décerner une médaille au maire qui «s'est exposé au danger pour terrasser un brigand $[. .$.$] serait d'un grand effet moral dans un pays où la justice éprouve$ souvent des difficultés pour l'arrestation des malfaiteurs ${ }^{48}$. Quinze ans plus tard, dans un contexte plus apaisé, le maire de Bordeaux adopte les mêmes accents pour rendre hommage au facteur Pouzalgues, qui a su freiner la fuite d'un voleur armé : «les dévouements de cette nature, malheureusement trop rares, demandent à être encouragés dans l'intérêt général ${ }^{49}$. Dans l'Aisne, enfin, c'est avec "un mouvement de curiosité sympathique» que le public des assises, d'ordinaire plutôt moqueur ${ }^{50}$, accueille la femme Desserville, qui a identifié un criminel en fuite dont elle a ainsi permis l'arrestation : «c'est à votre intelligence et à votre perspicacité que la société a dû d'être débarrassée d'un homme dangereux", la félicite le président du tribunal, qui invite la société à "prendre exemple» sur son dévouement ${ }^{51}$.

Le même élan pédagogique inspire Augustine Fouillée, qui prolonge son célèbre Tour de la France par Deux Enfants dans un autre manuel de lecture scolaire, Les Enfants de Marcel (1887). Le héros de cet ouvrage explique à son fils, qui a remarqué des ombres suspectes dans la cour, qu'il ne faut pas craindre d'intervenir : «Nous devons aide à nos voisins», dit-il avant de faire fuir les voleurs qui s'étaient introduits. En bon citoyen, il prévient ensuite la police, qui l'interroge et devant laquelle «il fait sa déposition avec la plus

46. Le pompier Gatineau est ainsi récompensé pour avoir lutté contre l'incendie à de nombreuses reprises, à quoi s'ajoute l'arrestation d'un forçat évadé; Arch.nat. F1dIII 389, mémoire récapitulatif des propositions pour une médaille d'honneur, année 1863.

47. Arch.nat. F1dIII 212, procès-verbal du commissaire de Saint-Amand, 15 avril 1878.

48. Arch.nat. F1dIII 230, rapport du sous-préfet de Mauléon au préfet des Basses-Pyrénées, 29 août 1863 .

49. Arch.nat. F1dIII 167, lettre du maire de Bordeaux au préfet de la Gironde, 15 février 1878.

50. Frédéric Chauvaud, La chair des prétoires. Histoire sensible de la cour d'assises, 1881-1932, Rennes, PUR, 2010, p. 156 et 180.

51. Arch.nat. F1dIII 102, dossier Césarine Desserville; Journal de l'Aisne, 16 et 17 novembre 1878. 
minutieuse exactitude, sans se préoccuper des commentaires auxquels elle pourrait donner lieu». Hélas le commissaire fait mine de se montrer suspicieux, pour le plus grand plaisir des esprits chagrins du voisinage : «c'est égal, ils auraient mieux fait de ne pas s'occuper de ce qui se passe là-bas, cela pourra bien leur attirer de l'ennui». Rumeur vite démentie : innocenté et félicité, Marcel éprouve surtout la satisfaction d'avoir donné «le bon exemple» à ses enfants ${ }^{52}$.

Par sa simple existence, ce chapitre prouve que la leçon ne va pas de soi : faut-il se mêler des affaires des autres? La question est au centre du Vérité d'Émile Zola, transposition à chaud de l'affaire Dreyfus en milieu scolaire. Héros du roman, l'instituteur Froment part en quête des témoins qui pourraient innocenter son collègue, accusé à tort. Mais tout le monde se tait : "Pourquoi donc les venait-on questionner ainsi? Ça ne regardait personne leurs idées. [...] Chacun son métier, les gendarmes savaient le leur». Ce n'est que dans l'avenir, conclut Zola, que Froment devenu vieux pourra assister à "ce spectacle nouveau, de simples citoyens apporter leur contribution, s'efforcer de dire tout ce qu'ils savaient ${ }^{53}$.

\section{De la COMmunauté Vigilante À la Foule CRIMinelle}

À la lecture des archives judiciaires, il convient toutefois de réévaluer la fonction policière du voisinage, aussi bien en ville, où subsistent encore longtemps des formes poussées de contrôle social ${ }^{54}$, qu’à la campagne. En cette seconde moitié du XIX ${ }^{e}$ siècle, le fait majeur est assurément le déclin des pratiques communautaires d'autorégulation, dont témoigne la folklorisation du charivari ou la moindre valeur des "arrangements", qui n'empêchent plus le recours à la justice ${ }^{55}$. On aurait toutefois tort d'en déduire que ces usages anciens ont complètement disparu - à plus forte raison dans des espaces inégalement policés. Insistons sur ce point : les gendarmes vivent dans de (petites) villes où ils exercent l'essentiel de leurs activités. Qu'ils soient réglementairement tenus de visiter au moins deux fois par mois chaque hameau de leur circonscription prouve précisément qu'ils n'y exercent qu'une surveillance distante, au mieux ponctuelle. Quand elle n'est pas confiée au garde champêtre, dont l'efficacité est rituellement contestée, la police quotidienne des villages reste donc encore souvent l'affaire des habitants eux-mêmes. De

52. G. Bruno [Augustine Fouillée], Les Enfants de Marcel, Paris, Belin, 1887, p. 158-177.

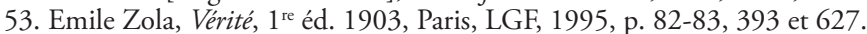

54. Alexandre Nugues-Bourchat, La Police et les Lyonnais au XIX' siècle. Contrôle social et sociabilité, Grenoble, PUG, 2010, p. 228, 296 et 308-316.

55. On en trouvera des exemples stimulants à travers l'histoire de l'infanticide ou de l'inceste : Annick Tillier, Des criminelles au village. Femmes infanticides en Bretagne (1825-1865), Rennes, PUR, 2001, p. 58; Fabienne Giuliani, Les liaisons interdites. Histoire de l'inceste au XIX' siècle, Paris, Publications de la Sorbonne, 2014, p. 199 et sq. Voir aussi Annick Le Douget, Violence au village, Rennes, PUR, 2014, p. 200. 
cette vigilance ordinaire, que les préfets ne pouvaient ou ne voulaient pas voir $^{56}$, les archives ne disent toutefois presque rien $^{57}$.

La disparition de la garde nationale ne semble pas avoir suscité de regrets ${ }^{58}$. Condamnée de longue date par les exigences de la professionnalisation ${ }^{59}$, elle paraissait tombée en désuétude dans les communes où elle subsistait. Il faut toutefois distinguer cette forme institutionnelle révolue de l'élan collectif qui avait pu lui donner consistance : «force sociale plus que politique, [...] la garde nationale fut largement un phénomène spontané " $"{ }^{60}$, rappelle Louis Girard, tandis que Maurice Agulhon évoque le «militarisme municipal diffus" qui constitue le socle commun des pratiques collectives de vigilance, des rondes de garde nationale ou des prises d'armes insurrectionnelles ${ }^{61}$.

Quelque chose de cet esprit communautaire persiste peut-être à la fin du $\mathrm{XIX}^{\mathrm{e}}$ siècle à travers le développement des associations de pompiers, encore trop méconnues, dont on sait toutefois qu'elles font l'objet, comme la garde nationale autrefois, d'une prise en main réglementaire qui a pu freiner l'ardeur militante des volontaires ${ }^{62}$. Moins institutionnalisé, le son du tocsin reste un puissant levier de mobilisation collective ${ }^{63}$. Sans qu'il soit nécessaire de réactiver la loi du 10 vendémiaire an IV sur la participation citoyenne à la lutte contre le brigandage, depuis longtemps tombée dans les oubliettes de la jurisprudence ${ }^{64}$, les villageois savent encore se rassembler dans le cadre de battues dans lesquelles le maire joue un rôle déterminant. En témoigne la "détermination héroïque» de Pierre Etcheberts, premier édile d'AinhiceMongelos (Basses-Pyrénées), qui s’attaque en 1862 aux brigands réfugiés dans sa commune : «après avoir réuni plusieurs individus qu'il posta à une distance de 400 mètres environ autour de [leur repaire], lui-même [s'y] diri-

56. Catherine Denys, «Policer les villages dans l'Europe des XVIII'-XIX ${ }^{e}$ siècles», in Laurent Brassart, Jean-Pierre Jessenne et Nadine Vivier [dir], Clochemerle ou république villageoise? La conduite municipale des affaires villageoises en Europe, Lille, Presses du Septentrion, p. 307-316.

57. Arnaud-Dominique Houte, "Que faire quand on est volé? Porter plainte dans la France rurale du XIX ${ }^{\mathrm{e}}$ siècle», in Martine Charageat et Mathieu Soula [dir], Dénoncer le crime du Moyen-Âge au XIXesiècle, Talence, MSHA, 2014, p. 317-328.

58. La postérité de la garde nationale est esquissée par Georges Carrot, La garde nationale, une force publique ambiguë, 1789-1871, Paris, L'Harmattan, 2003, p. 325. Quelques pistes et beaucoup d'outils dans Serge Bianchi et Roger Dupuy [dir.], La Garde nationale entre nation et peuple en armes. Mythes et réalités, 1789-1871, Rennes, PUR, 2006.

59. Selon un modèle dont on retrouve les racines au XVIII siècle : Marco Cicchini, «Milices bourgeoises et garde soldée à Genève au XVIII siècle. Le républicanisme classique à l'épreuve du maintien de l'ordre", Revue d'histoire moderne et contemporaine, avril-juin 2014, p. 120-149.

60. Louis Girard, La Garde nationale, 1814-1871, Paris, Plon, 1964, p. 361.

61. Maurice Agulhon, La République au village, Paris, Plon, 1970, p. 453.

62. Alan R. Baker, Fraternity among the French Peasantry: Sociability and Voluntary Associations in the Loire Valley, 1815-1914, Cambridge, CUP, 1999, notamment p. 218.

63. Alain Corbin, Les Cloches de la terre. Paysage sonore et culture sensible dans les campagnes au XIX'e siècle, Paris, Albin Michel, 1994, p. 192.

64. Aurélien Lignereux, La France rébellionnaire..., op. cit., p. 314. Les formes de la battue s'inspirent davantage du moule cynégétique, encore très vivace; Jean-Marc Moriceau, L'homme contre le loup. Une guerre de deux mille ans, Paris, Fayard, 2011, p. 243 et 310. 
gea avec un de ses compagnons à qui il avait fait jurer de ne pas l'abandonner ${ }^{65}$.

Accordons une place à part à l'arrestation de Sylvain Poirier, à laquelle Jean-Claude Farcy a consacré une belle monographie. La traque de cet assassin en fuite mobilise, au printemps 1874, une dizaine de gendarmes et environ 700 villageois « réunis sous la direction des magistrats et espacés comme des tirailleurs» : «tous les hommes de bonne volonté étaient priés de se réunir à neuf heures du matin sur la place publique. Il leur était permis de s'armer, mais il leur était spécialement recommandé de ne pas se servir de leurs armes destinées seulement à effrayer le coupable et à empêcher le percement des lignes", résume le procureur ${ }^{66}$. Atypique par son ampleur, le procédé passionne Le Figaro, qui décrit la fureur de la foule et son envie de meurtre. Calomnies, réplique l'instituteur du village : «La loi de Lynch s'applique en Amérique, mais tous ces braves gens savaient que le meurtrier n'appartenait qu'à la justice et n'ont cherché qu'à le lui remettre vivant. Certes le coupable a subi toutes les humiliations de cette foule, mais personne n'eut l'intention de lui ravir la vie» ${ }^{67}$.

Entre la mobilisation citoyenne et le lynchage, la frontière semble nécessairement poreuse ${ }^{68}$. Pour le seul premier semestre 1897, les rapports des procureurs généraux recensent ainsi une bonne dizaine de regroupements plus ou moins menaçants. Ce sont le plus souvent des habitants indignés qui s'attroupent au passage d'un criminel, le huent et déstabilisent parfois les forces de l'ordre ${ }^{69}$. Si ces débordements de colère peuvent déboucher sur des dégradations et des violences ${ }^{70}$, ils restent sous contrôle et n'inquiètent guère les pouvoirs publics, qui n'y voient que l'expression des passions locales et des structures communautaires de voisinage. Dans les cas les plus graves, le discours des autorités rejette la faute sur les éléments étrangers : les Lyonnais n'ont rien à voir avec les pogroms anti-italiens, explique ainsi le préfet du Rhône, qui accuse «une tourbe d'émeutiers dont les honnêtes gens réprouvent les méfaits ${ }^{71}$. À Aigues-Mortes, où le lynchage s'achève en tuerie, la justice poursuit les «trimards» et oublie les encouragements qui leur furent prodigués, plus ou moins directement, par les propriétaires de vignes ${ }^{72}$.

65. Arch.nat. F1dIII 230, lettre de l'adjoint au maire d'Ainhice-Mongelos au préfet des BassesPyrénées, 11 août 1862. Le maire obtient une médaille d'argent pour récompense de son courage.

66. Arch.nat. BB ${ }^{24}$ 2040/1, rapport du procureur général, 3 septembre 1874.

67. Journal de Chartres, 11 juin 1874, cité par Jean-Claude Farcy, Meurtre au bocage. L'affaire Poirier (1871-1874), Chartres, SAEL, 2012, p. 242.

68. La gradation des attitudes de la foule est au centre des analyses pionnières de William $\mathrm{F}$. Brundage, Under Sentence of Death : Lynching in the South, Chapel Hill, UNCP, 1997.

69. Arch.nat. BB ${ }^{18}$ 2050, rapport du procureur d'Amiens, 5 février 1897.

70. Arch.nat. $\mathrm{BB}^{18}$ 2295, affiche du maire de Belfort, 3 juin 1897; rapport du procureur de Besançon, 9 juin 1897.

71. Arch.nat. $\mathrm{F}^{7} 12512$, rapport du préfet du Rhône au ministre de l'Intérieur, 26 juin 1894. Voir aussi Karine Salomé, Je prie pour Carnot qui va être assassiné ce soir. Un attentat contre la République, 24 juin 1894, Paris, Vendémiaire, 2012, p. 63-70.

72. Gérard Noiriel, Le massacre des Italiens. Aigues-Mortes, 17 août 1893, Paris, Fayard, 2010, p. 45, 59, 67-68. 
Le modèle américain du lynchage, c'est-à-dire la volonté des habitants de se substituer à une police et à une justice défaillantes ${ }^{73}$, n'existerait donc pas en France. Fascinés, à la suite des récits de Maxime Du Camp et d'Hippolyte Taine, par les violences de foule ${ }^{74}$, les criminologues de la fin du XIX ${ }^{\mathrm{e}}$ siècle considèrent en tout cas que les scènes de lynchage sont "un phénomène typiquement américain, inconnu heureusement en Europe $»^{75}$. Il s'agit, explique Arthur Desjardins, d'un "grave désordre" qui remet en cause «les principes élémentaires de la civilisation ${ }^{76}$. "Même appliquée sans erreur, la loi du juge Lynch est une loi que la raison et l'humanité condamnent", clame à son tour Le Petit Parisien. Signe des temps, le grand quotidien précise toutefois, en 1901 , qu' «il faut le répéter souvent ${ }^{77} \ldots$

\section{CRISE SÉCURITAIRE ET MOBILISATION SOCIALE}

On sait que la société française connaît, au tournant du siècle, une crise sécuritaire qui s'explique en grande partie par l'exposition médiatique de la criminalité, mais qui procède aussi des transformations qualitatives et quantitatives de la délinquance et, davantage encore, d'une perte de confiance à l'égard de l'institution policière. Fait suggestif, bien que difficile à interpréter : la part des plaintes directement transmises par les particuliers au parquet se redresse (10\% de l'ensemble des plaintes en 1890, $20 \%$ en 1910), après un siècle de recul continu : les citoyens s'en remettraient-ils moins naturellement aux professionnels de l'ordre ${ }^{78}$ ? Admettons du moins qu'ils leur accordent moins de crédit, tant en ville qu'à la campagne, où la découverte des crimes de Vacher, à l'automne 1897, fait l'effet d'un choc et provoque l'ouverture d'une commission extraparlementaire sur la sécurité rurale ${ }^{79}$. C'est dans ce nouveau contexte qu'est remise en discussion la participation populaire à la sécurité publique.

La question se pose dès le printemps 1892, dans une société parisienne perturbée par une vague d'attentats anarchistes. On l'a dit, le pays se divise sur le cas Lhérot. «Faites votre police-mêmes, camarades», encourage à son

73. Tangi Villerbu, «Retrouver les origines du lynchage : violence, peuple et justice dans la jeune République américaine", in Frédéric Chauvaud et Pierre Prétou [dir.], op. cit., p. 181-186; Joël Michel, Le lynchage aux États-Unis, Paris, La Table Ronde, 2008, p. 122 et sq.

74. Susanna Barrows, Miroirs déformants. Réflexions sur la foule en France à la fin du XIXe siècle, Paris, Aubier, 1990; Martine Kaluszynski, La République à l'épreuve du crime. La construction du crime comme objet politique, 1880-1920, Paris, LGDJ, 2002, p. 110-112; Olivier Bosc, La foule criminelle. Politique et criminalité dans l'Europe du tournant du XIX $X^{e}$ siècle, Paris, Fayard, 2007.

75. Scipio Sighele, La foule criminelle, Paris, Alcan, 1892, p. 160.

76. Arthur Desjardins, "Le droit des gens et la loi de Lynch aux USA», Revue des Deux Mondes, mai 1891 , p. 321-355.

77. «Les justiciers», Le Petit Parisien, 3 juillet 1901.

78. Jean-Claude Farcy, «La gendarmerie, police judiciaire au XIX" siècle», Histoire Économie et Société, 2001, n 3, p. 385-403.

79. Arnaud-Dominique Houte, Le métier de gendarme..., op. cit., p. 267. 
tour Le Matin, qui appelle les ouvriers à se mobiliser contre les anarchistes. Au risque de la "guerre civile sous forme d'une contre-chasse à la dynamite", s'inquiète Marcel Schwob : "on croirait que nous sommes au Texas ${ }^{80}$. Fautil prendre ces témoignages au pied de la lettre? Est-il vrai, comme l'écrit Zola, que "certains [Parisiens] allaient jusqu'à faire une enquête personnelle pour mettre eux-mêmes la main sur les coupables ${ }^{81}$ ? Il est permis d'en douter : en dehors d'une épidémie de lettres anonymes, rien de significatif n'émerge des archives, pourtant copieuses ${ }^{82}$.

Le changement de ton est toutefois notable et pourrait être mis en relation avec un regain des formes de surveillance citoyenne, signalé et salué par La Lanterne dès le printemps $1893^{83}$. Prenons l'exemple du cambriolage des locaux de "L'Urbaine», à Saint-Ouen, dont on apprend par la presse qu'il a été déjoué par quatre commerçants du voisinage armés et attentifs aux mouvements suspects de la rue ${ }^{84}$. Ces "paisibles boutiquiers" improvisés en "apprentis Lecoq" amusent davantage qu'ils n’intéressent la journaliste Séverine ${ }^{85}$, mais leur mobilisation semble s'insérer dans un mouvement plus global qui gagne sans doute davantage de visibilité que de consistance au début $\mathrm{du} \mathrm{XX}^{\mathrm{e}}$ siècle. Tandis que les techniques d'autodéfense gagnent en audience ${ }^{86}$ et que le port d'armes se banalise ${ }^{87}$, les formes d'organisation collectives se multiplien - ou se font plus ostensiblest. Le Petit Parisien cite ainsi les habitants de Saint-Mandé, qui se liguent contre une bande qui sévit dans le bois de Vincennes : «ceux qui rentrent tard doivent compter beaucoup sur leurs propres forces; il est donc prudent pour eux de s'armer et d'user de leurs armes sans hésitation contre ces jeunes malfaiteurs " ${ }^{88}$. À Colombes, c'est en 1905 qu'est fondée, par le journaliste Jacques Dhur, une «ligue de défense» qui se charge de "protéger les habitants des communes suburbaines " ${ }^{89}$. Deux ans plus tard, une société des "Détectives Parisiens " prévoit d'instituer, à côté

80. Marcel Schwob, "Lettres Parisiennes", Le Phare de la Loire, 30 avril 1892, repris dans Chroniques, Genève, Droz, 1981.

81. Émile Zola, Les trois villes : Paris, Paris, Nouveau Monde, 2008 [1897], p. 129.

82. Des sondages infructueux ont été faits dans les fonds des Archives Nationales et des Archives de la préfecture de police qui avaient déjà été repérés et fouillés par Jean Maitron (op. cit.).

83. La Lanterne, 26 mars 1893.

84. Arch. dép. Paris, D5U9 121, Chambre des mises en accusation, séance du 18 mars 1893.

85. Séverine, «Pour un lapin!», dans En marche, Paris, Simonis, 1896, p. 85-88.

86. Aaron Freundschuh, "New Sport in the Street: Self-Defence, Security and Space in Belle Epoque Paris", French History, 2006-4, p. 424-441.

87. Dominique Kalifa, L'Encre et le Sang..., op. cit. La miniaturisation croissante des armes de poing inquiète cependant les pouvoirs publics, comme le montre un copieux dossier constitué en 1912-13 dans les cartons de la chancellerie (Arch.nat. BB ${ }^{18}$ 6538). Par la circulaire du 20 août 1912, le garde des Sceaux stimule l'ardeur répressive de ses procureurs, mais leur rappelle que «l'indulgence s'impose à l'égard de l'honnête homme trouvé porteur d'une arme prohibée, mais ayant pour excuse la menace d'une agression ». Le procureur d'Angers explique ainsi, à propos de la clémence dont profite un homme armé d'un browning chargé : «il a eu le tort de sortir de chez lui avec un revolver dont la présence à son domicile se justifiait pleinement» (14 mai 1913, dossier Portier). De manière générale, ceux qui peuvent s'honorer de «bons renseignements de moralité» encourent, au pire, l'amende - le plus souvent avec sursis - et la confiscation de leur arme.

88. «La bande de Saint-Mandé», Le Petit Parisien, 17 janvier 1899.

89. Le Journal, 9 juillet 1905. 
d'une agence de police privée, "une brigade d'amateurs" qui aurait pour mission de "surveiller attentivement et intelligemment tout ce qui se passe d'illicite sur la voie publique $»^{90}$. En 1908, enfin, émerge une "ligue de la sécurité publique» qui entend, de la même manière, "organiser des compagnies de volontaires pour assurer la sécurité de la rue et des campagnes ${ }^{91}$.

Pratiques d'autodéfense et scènes de lynchage tiennent le devant de la scène médiatique ${ }^{92}$, sans que l'on puisse déterminer si ces actes sont réellement plus fréquents ou s'ils sont seulement mis en relief. Toujours est-il que la perception du phénomène s'infléchit nettement. En pointe du mouvement, Le Petit Parisien ne s'émeut plus des graves blessures subies par des apaches qui s'étaient attaqué à un passant armé d'un browning : «les rôdeurs trouvent à qui parler». Il se réjouit de la même manière de l'arrestation brutale d'un vieux pervers : " on lui donna la chasse, la foule s'ameuta, le cerna et ayant bien vite appris l'attentat qu'on lui reprochait, elle lui fit passer un bien mauvais quart d'heure " ${ }^{93}$. Ritualisés, ces récits trouvent une transcription burlesque à travers les premières aventures des Pieds-Nickelés : Croquignol, Ribouldingue et Filochard sont toujours repérés par la foule, principalement paysanne et toujours "armée de fourches et de bâtons", qui se met à leur poursuite - «filous, voleurs, crapules!» - et leur administre "une pâtée en règle» aux allures de lynchage ${ }^{94}$.

Les formes de ce raidissement sécuritaire inspirent une lecture critique qui s'exprime sous la plume pourtant répressive du procureur général de Lyon, Loubat: «il n'est pas nécessaire d'armer les honnêtes gens pour désarmer les apaches». Du reste, ajoute-t-il, «les braves gens tiennent-ils tant que cela au droit au revolver? "95. La dénonciation des violences de l'ordre est plus mordante dans L'Assiette au Beurre, où sévit le trait acéré de Félix Valloton : «Tu y reviendras, cochon, pisser sur mon mur!», s'exclame un bourgeois qui fait feu sur un passant. "Tu la trouves un peu dure celle-là», conclut un cultivateur qui vient d'abattre un chapardeur de fruits ${ }^{96}$. Ces dessins pourraient illustrer le discours du député socialiste Victor Dejeante, qui s'élève contre

90. La Presse, 6 janvier 1907. Les statuts des "Détectives Parisiens» sont reproduits par Dominique Kalifa, Naissance de la police privée. Détectives et agents de recherche en France, 1832-1942, Paris, Perrin, 2000, p. 289.

91. A. Rivière, «Défendons-nous», Revue pénitentiaire, 1909, p. 282-284.

92. Par exemple Le Petit Parisien, 25 juillet et 24 novembre 1910. Voir Dominique Kalifa, L'encre et le sang..., op. cit., p. 261-264; Anne-Claude Ambroise-Rendu, "L'infrajudiciaire dans les récits de faits divers : la foule, justicière ou meurtrière? (1870-1910)", dans B. Garnot [dir], Linfrajudiciaire du Moyen-Âge à l'époque contemporaine, Dijon, EUD, 1996, p. 447-456.

93. Le Petit Parisien, 10 avril 1912 et 17 novembre 1908. D'autres affaires du même type sont signalées, le 17 novembre 1908 et le 3 mars 1913.

94. La bande des Pieds-Nickelés, première série d'aventures, Montrouge, De Varly Editions, 2012 ( $1^{\text {re }}$ édition 1908$)$, p. $10,30-31,33,38$ et 65.

95. Guillaume Loubat, «L'abus du revolver», texte publié dans Le Matin en 1910; Arch.nat. BB ${ }^{18}$ 6541. On sait que la mise en place d'une garde civile, en 1914, achoppe précisément sur le refus de la plupart des citoyens de s'armer à leurs frais.

96. "Crimes et châtiments", L'Assiette au Beurre, $1^{\mathrm{er}}$ mars 1902. 
l'importation en France de "cette cruelle et lâche loi de Lynch " ${ }^{97}$. Certains exhument même le spectre de la "Société des gourdins réunis", du nom d'une éphémère organisation fondée au lendemain des grandes manifestations républicaines de février $1870^{98}$ : critiquant la «torpeur blâmable» de la bourgeoisie, le directeur du Figaro, Hippolyte de Villemessant, avait alors proposé de constituer «une armée de 40000 honnêtes gens" déterminés à "opposer la manifestation de l'ordre à la manifestation du désordre " ${ }^{99}$. On s'était beaucoup moqué, à l'époque, de ces "compagnons de la trique» qui prétendaient "remplacer la gendarmerie » 100 et dont l'existence concrète était restée très limitée ${ }^{101}$. C'est avec le même mélange d'ironie et d'indifférence que les autorités publiques semblent accueillir ces nouvelles initiatives dont la portée pratique semble tout aussi réduite.

«Les habitants devraient faire la police eux-mêmes et ne pas compter sur les gendarmes", avance néanmoins le député radical Alexandre Boutard, lors d'un débat sur la situation inquiétante de Longwy, cette "seconde Calabre»; "c'est ce qu'ils sont obligés de faire déjà, mais avouez que ce n'est pas là une situation normale", réplique Albert Lebrun, qui sollicite - et obtient - des renforts de gendarmerie ${ }^{102}$. Par-delà l'enjeu budgétaire, cet échange révèle aussi la survie du vieil imaginaire révolutionnaire de la mobilisation civique, dont le philosophe Alain se montre le plus éloquent défenseur : "Si nous vivions démocratiquement, il est à croire que chacun participerait de sa personne à la fonction de police», que la société a trop facilement délégué à "une espèce de garde prétorienne». "Comme on exige de tout citoyen qu'il porte de l'eau à l'incendie, ainsi on peut exiger que tout citoyen fasse méthodiquement la police autour de lui », conclut-il en rêvant d'un "encadrement patient" des passions policières qui s'expriment par les réactions désordonnées de la foule ${ }^{103}$.

Comme pour lui donner raison, l'affaire Bonnot, paroxysme de la crise sécuritaire, se solde par la mise en scène d'une véritable mobilisation générale : "Soldats, agents, inspecteurs, simples citoyens sans mandat, tous, à l'heure voulue, ont marché contre le bandit», se félicite Le Matin, tandis que des cartes postales mettent en scène "gardes municipaux et habitants

97. Débats de la Chambre des députés, 3 juillet 1908.

98. Fabien Cardoni, «Entre rétablissement et maintien de l'ordre : la résolution des conflits parisiens de 1868 à 1870 ", dans Jean-Claude Caron, Frédéric Chauvaud, Emmanuel Fureix et JeanNoël Luc [dir], Entre violence et conciliation. La résolution des conflits sociopolitiques en Europe au XIX'siècle, Rennes, PUR, 2008, p. 117-126.

99. «La société des gourdins réunis», Le Figaro, 11 février 1870.

100. Le Gaulois, 16 février 1870; Pierre Barbier et France Vernillat, Histoire de France par les chansons. La République de 1848 et le Second Empire, Paris, Gallimard, 1959, p. 164-166.

101. «Au premier désordre, les membres de cette société se contenteront de s'enfermer dans leur cave en se servant de leurs gourdins pour faire du feu ", jugeait Le Gaulois (13 février 1870). L'association est toutefois citée à propos de la manifestation bourgeoise du 23 mars 1871, au tout début de la Commune.

102. Débats de la Chambre des députés, 2 mars 1906; voir aussi Le Gendarme, 1er avril 1906

103. La Dépêche de Rouen et de Normandie, 9 avril et 28 avril 1912, repris dans Alain, Les Propos d'un Normand de 1912, Paris, Klincksieck, 1998, p. 137-138 et 161-162. 
tirant sur la maison" du bandit, à Choisy-le-Roi ${ }^{104}$. À lire plus attentivement la presse, on s'aperçoit toutefois bien vite que les «francs-tireurs» de l'ordre public n'ont joué qu'un rôle dérisoire, comme le confirme, quinze jours plus tard, l'assaut dirigé contre les derniers rescapés de la bande, Garnier et Valet, réfugiés à Nogent-sur-Marne. Là aussi, «un habitant s'offre à pénétrer avec son chien; il entre, couvert par un bouclier, le revolver à la main». Non seulement l'opération est un échec, mais elle tourne au ridicule quand le chien s'attaque aux mollets policiers, pour le plus grand amusement des journalistes $^{105}$ - bon résumé, somme toute, des impasses d'une mobilisation populaire bien plus médiatique qu'efficace.

Comme les sociologues l'ont montré pour des périodes récentes ${ }^{106}$, la participation populaire aux fonctions de police s'inscrit en grande partie dans les cadres communautaires du voisinage dont elle épouse les hiérarchies et les préjugés. Survivance archaïque d'un ordre social bientôt révolu, elle n'en fait pas moins partie, à un autre niveau, du programme civique républicain, et elle participe à cet égard à la construction et à la préservation du nouvel ordre démocratique. De même que le jury d'assises rappelle l'utopie révolutionnaire d'une justice populaire, la figure du "citoyen-policier" peut ainsi contrebalancer l'emprise de plus en plus exclusive qu'exerce l'État sur les forces de l'ordre.

Encore riche de multiples potentialités dans la seconde moitié du $\mathrm{XIX}^{\mathrm{e}}$ siècle, cet imaginaire semble néanmoins s'affadir, ramené à sa seule dimension sécuritaire. Il suscite surtout la méfiance croissante des autorités publiques, qui ne jurent plus que par le modèle d'un ordre professionnalisé. Leur inquiétude se cristallise durant le premier mois de la guerre : tandis que des foules vengeresses engagent une furieuse chasse aux espions ${ }^{107}$, les "policiers de bonne volonté» qui s'organisent au sein des "gardes civiles» se signalent par leurs piètres résultats qui aboutissent à l'arrêt précipité de l'expérience ${ }^{108}$. L'échec témoigne de la perte de sens d'un "citoyen-policier» disqualifié, à plus forte raison, par le succès de représentations critiques - et

104. Le Matin, 30 avril 1912. Une riche série de cartes postales de la collection Zoumeroff a été mise en ligne sur le portail Criminocorpus.

105. Le Petit Journal, 16 mai 1912; Laurent Lopez, La guerre des polices n'a pas eu lieu. Gendarmes et policiers coacteurs de la sécurité publique sous la Troisième République, 1870-1914, Paris, PUPS, 2014, p. 382 et $s q$.

106. Avant de rebondir au début du XXI e siècle, le débat sur la sécurité citoyenne avait connu un temps fort à la fin des années 1970; Renaud Dulong, L'autodéfense. Enquête sur quelques faits indécidables, Paris, Librairie des Méridiens, 1983. Depuis 2015, la question du vigilantisme fait l'objet d'un séminaire, animé par Gilles Favarel-Garrigues et Laurent Gayer, à Sciences-Po (CERI-CNRS).

107. Connu, le phénomène est réévalué par Bruno Cabanes, Aoùt 14, la France entre en guerre, Paris, Gallimard, 2014, p. 145 et 180.

108. Arnaud-Dominique Houte, «Policiers de bonne volonté? L'impossible constitution d'une garde civile en France (1913-1920)», Vingtième Siècle, avril-juin 2013, p. 159-170. 
l'on se permettra de conclure ici en citant Jacques Prévert, dont la célèbre "Chasse à l'enfant» scelle l'émergence d'un nouvel imaginaire :

«Et tous galopent après lui

Les gendarmes les touristes les rentiers les artistes

Bandit! Voyou! Voleur! Chenapan!

C'est la meute des honnêtes gens

Qui fait la chasse à l'enfant

Pour chasser l'enfant pas besoin de permis

Tous les braves gens s'y sont mis. ${ }^{109}$

Arnaud-Dominique Houte est maître de conférences à Paris-Sorbonne et membre du Centre de Recherche en Histoire du XIXe siècle (Paris I-Paris IV) 